\title{
On collective complete integrability according to the method of Thimm
}

\author{
VICTOR GUILLEMIN AND SHLOMO STERNBERG \\ Department of Mathematics, Massachusetts Institute of Technology, Cambridge, \\ Massachusetts, 02139, U.S.A.; Department of Mathematics, Harvard University, \\ Cambridge, Massachusetts, 02138, U.S.A.
}

(Received 27 June 1982 and revised 14 January 1983)

\begin{abstract}
Let $G$ be a Lie group acting in Hamiltonian fashion on a symplectic manifold $M$ with moment map $\Phi: M \rightarrow g^{*}$. A function of the form $f \circ \Phi$ where $f$ is a function on $g^{*}$ is called 'collective'. We obtain necessary conditions on the $G$ action for there to exist enough Poisson commuting functions on $g^{*}$ so that the corresponding collective functions on $\boldsymbol{M}$ form a completely integrable system. For the case $G=O(n)$ or $U(n)$ these conditions are sufficient. This explains Thimm's proof [17] of the complete integrability of the geodesic flow on the real and complex grassmanians. We also discuss related questions in the geometry of the moment map.
\end{abstract}

\section{Introduction}

In a recent paper [17], Thimm has devised a method for proving the complete integrability of a large collection of interesting Hamiltonian systems. His method, roughly speaking, is as follows. Suppose we are given a Hamiltonian action of a Lie group $G$ on a symplectic manifold $M$ with attendent moment map

$$
\Phi: M \rightarrow g^{*}
$$

(cf. [3] for the relevant definitions). If $H$ is a $G$-invariant Hamiltonian it commutes with all functions of the form

$$
f \circ \Phi \quad f: g^{*} \rightarrow \mathbb{R} .
$$

(Such functions are called 'collective' in [3] because of their relevance to various models of the nucleus.) The space of smooth functions on $\mathrm{g}^{*}$, which we denote by $\mathscr{F}\left(g^{*}\right)$, has its own Poisson bracket, the Kostant-Souriau bracket which we shall denote by \{\}$_{g^{*}}$. We denote the Poisson bracket on $M$ by $\{,\}_{M}$ and recall from [3] that the map

$$
f \rightsquigarrow \Phi^{*} f=f \circ \Phi
$$

is a homomorphism for the Lie bracket structures, i.e., that

$$
\Phi^{*}\left\{f_{1}, f_{2}\right\}_{g^{*}}=\left\{\Phi^{*} f_{1}, \Phi^{*} f_{2}\right\}_{M} .
$$

If $f_{1}$ is a $G$-invariant function, then

$$
\left\{f_{1}, f_{2}\right\}_{g^{*}}=0 \quad \text { for all } f_{2}
$$


and hence if $f_{1}, \ldots, f_{r}$ are independent $G$-invariant functions, the functions

$$
\Phi^{*} f_{1}, \ldots, \Phi^{*} f_{r}
$$

will all Poisson commute on $M$ and also Poisson commute with any $G$ invariant $H$. Of course, in general, there will not be enough of them. But suppose that we consider a subalgebra $g_{1} \subset g$. This gives rise to a projection

$$
g^{* \pi} \rightarrow g_{1}^{*}
$$

and hence a homomorphism

$$
\mathscr{F}\left(\mathrm{g}_{1}^{*}\right) \stackrel{\pi^{*}}{\rightarrow} \mathscr{F}\left(\mathrm{g}^{*}\right)
$$

of Poisson algebras. If we now consider the invariants $f_{1}^{1}, \ldots, f_{r_{1}}^{1}$ in $\mathscr{F}\left(g_{1}^{*}\right)$, they Poisson commute with one another, and thus

$$
\pi^{*} f_{1}^{1}, \ldots, \pi^{*} f_{r_{1}}^{1}
$$

Poisson commute with one another and with the invariants of $g$. Hence pulling them back to $M$ via $\Phi$ gives more Poisson commuting integrals of $H$.

(We point out that the Poisson bracket of two functions on $g^{*}$ can be defined as follows: at any point, $x \in g^{*}$, restrict the functions to the $G$ orbit $\mathcal{O}$ through $\alpha$. All $G$ orbits are symplectic manifolds. Then at $\alpha$,

Also the map

$$
\left\{f_{1}, f_{2}\right\}_{g^{*}}=\left\{f_{1}, f_{2}\right\}_{0} \text {. }
$$

$$
\left.\pi^{*}\right|_{0}: 0 \rightarrow g_{1}^{*}
$$

is the moment map for the $G_{1}$ action on $\mathcal{O}$. Thus the passage from $f_{1}^{1}$ to $\pi^{*} f_{1}^{1}$ is essentially the same as the passage from $f$ to $\Phi^{*} f$.) Proceeding in this way we can consider a chain

$$
g \supset g_{1} \supset \cdots \supset g_{k}
$$

of subalgebras and iteratively pull back the invariants. Under certain circumstances this will lead to $n / 2$ independent integrals where $n=\operatorname{dim} M$, i.e., to complete integrability. This is Thimm's method. In a sense it is a generalization of the Gelfand-Ceitlin method described in [3, p. 280]

The purpose of this note is to investigate when this method can be expected to work, to give some alternative proofs of the complete integrability of some of the examples on Thimm's list and to add some new classes of examples. We will also construct, by a related procedure, an entirely new class of integrable systems. These examples are obtained by considering the pre-image under the moment map of a section to the coadjoint $G$ action.

As a preliminary we need to develop some extensions of known facts about the moment map which have an interest in their own right.

\section{Generalities about the moment map}

Let $\Phi: M \rightarrow g^{*}$ be the moment map of a Hamiltonian $G$ action. For each $p \in M$ the $G$ action defines a linear map

$$
u_{p}: g \rightarrow T M_{p}
$$


and the symplectic form at $p$ gives an isomorphism of $T M_{p}$ with $T M_{p}^{*}$. We denote the composite of these two maps by $\psi_{p}$ so that

$$
\psi_{p}: g \rightarrow T M_{p}^{*}
$$

On the other hand we have the differential of the moment map at $p$

$$
d \Phi_{p}: T M_{p} \rightarrow g^{*} \text {. }
$$

The basic formula in this whole subject says that these two maps are transposes of one another:

$$
\left(d \Phi_{p}\right)^{*}=\psi_{p}
$$

cf. [5, formula (3.2)] or [4, after formula $\left.(2.7)^{\prime \prime}\right]$.

Suppose that the image of $M$ under $\Phi$ is a submanifold, $W$, of $g^{*}$ and that

$$
d \Phi_{p}\left(T M_{p}\right)=T W_{\alpha}
$$

where $\alpha=\Phi(p)$. Then (1.1) implies that

$$
\operatorname{ker} \psi_{p}=T W_{\alpha}^{0}
$$

the annihilator of $T W_{\alpha}$ in $g$. But $\operatorname{ker} \psi_{p}=g_{p}$, the isotropy algebra of $p$. Thus

$$
g_{p}=T W_{\alpha}^{0} \quad \alpha=\Phi(p) .
$$

Now since $\Phi$ is equivariant we clearly have

$$
g_{p} \subset g_{\alpha} \text {. }
$$

Let $G_{\alpha}$ be the isotropy group of $\alpha$. Clearly $G_{\alpha}$ leaves $W$, and hence $T W_{\alpha}$, invariant. Therefore $G_{\alpha}$ preserves $T W_{\alpha}^{0}$. Thus

$$
g_{p} \text { is invariant under the adjoint representation of } G_{\alpha}
$$

in particular

$$
g_{p} \text { is an ideal in } g_{\alpha} \text {. }
$$

Let $\sigma$ denote the $G$-orbit through $\alpha$, so $\sigma \subset W$. It is known cf. [8], and is an immediate consequence of (1.1) that $\Phi^{-1}(\mathcal{O})$ is a co-isotropic submanifold, and that the null foliation through $p$ of $\Phi^{-1}(\mathcal{O})$ is swept out by the connected component of $G_{\alpha}$. Indeed, it follows from (1.1) that

$$
\operatorname{ker} d \Phi_{p}=u_{p}(g)^{\perp}
$$

where $\perp$ denotes perpendicular relative to the symplectic form on $T M_{p}$. Since $\Phi$ is equivariant,

$$
d \Phi_{p}\left(u_{p}(g)\right)=T O_{\alpha} \quad \alpha=\Phi(p)
$$

and hence, since $\Phi: M \rightarrow W$ is clean,

$$
d \Phi_{p}^{-1}\left(T \mathcal{O}_{\alpha}\right)=u_{p}(g)+u_{p}(g)^{\perp} .
$$

Its null space is

$$
u_{p}(g) \cap u_{p}(g)^{\perp} \subset u_{p}(g)+u_{p}(g)^{\perp}
$$

so $d \Phi_{p}^{-1}\left(T O_{\alpha}\right)$ is co-isotropic. Also, by (1.6), if $\xi \in g$ then $u_{p}(\xi) \in u_{p}(g)^{\perp}$ if and only if $\xi \in g_{\alpha}$. Thus

$$
d \Phi_{p}^{-1}\left(T O_{\alpha}\right)^{\perp}=u_{p}(g) \cap u_{p}(g)^{\perp}=u_{p}\left(g_{\alpha}\right)
$$


Let $G_{\alpha}^{0}$ and $G_{p}^{0}$ denote the connected components of $G_{\alpha}$ and $G_{p}$. Since $G_{p}$ and $G_{\alpha}$ are given as isotropy subgroups it follows that $G_{p}^{0}$ is a closed subgroup of $G_{\alpha}^{0}$ which is a closed subgroup of $G$. From (1.8) we see that $G_{\alpha}^{0} \cdot p$ is the null foliation through $p$. It follows from (1.5) that

$$
G_{p}^{0} \text { is a normal subgroup of } G_{\alpha}^{0}
$$

and hence, since $G_{a p}=a G_{p} a^{-1}$, it follows from (1.9) that

$$
G_{p^{1}}^{0}=G_{p}^{0} \quad \text { for any } p^{1} \text { on } L_{p},
$$

where $L_{p}$ is the leaf of the null foliation of $\Phi^{-1}(\mathcal{O})$ through $p$. It also follows immediately from the preceding discussion that

$$
G_{\alpha}^{0} / G_{p}^{0} \text { acts locally freely on } L_{p} \text {. }
$$

We will now show that

(1.12) If $\operatorname{dim} O$ is maximal among all $G$ orbits in $W$ then $G_{\alpha}^{0} / G_{p}^{0}$ is abelian.

This is a generalization of a theorem of Duflo and Vergne [2] which asserts that if $\mathcal{O}$ is a maximal dimensional orbit in $g^{*}$ and $\alpha \in \mathcal{O}$ then $g_{\alpha}$ is abelian. The result (1.12) was obtained independently by Mishchenko in [12].

Proof of (1.12). From the definition of $g_{\alpha}$ it follows that if $\xi \in g_{\alpha}$ then

$$
\langle\alpha,[\xi, \zeta]\rangle=0 \quad \text { for all } \zeta \in g,
$$

where $\langle$,$\rangle denotes the pairing between g^{*}$ and $g$. If $\operatorname{dim} O$ is maximal, this means that $\operatorname{dim} g_{\beta}$ is locally constant near $\alpha$ and hence the $g_{\beta}$ fit together to form a smooth vector bundle over $W$ near $\alpha$. Thus, given any smooth curve $\alpha_{t}$ in $W$ with $\alpha_{0}=\alpha$ and any $\xi$ and $\eta \in g_{\alpha}$, we can find smooth curves $\xi_{t}$ and $\eta_{t}$ where

and

$$
\xi_{t} \in g_{\alpha_{t}}, \quad \eta_{t} \in g_{\alpha_{t}}
$$

Then

$$
\xi_{0}=\xi, \quad \eta_{0}=\eta
$$

$$
\left\langle\alpha_{t},\left[\xi_{t}, \eta_{t}\right]\right\rangle \equiv 0
$$

Let denote derivative at $t=0$. Then differentiating the preceding equation gives

$$
0=\langle\dot{\alpha},[\xi, \eta]\rangle+\langle\alpha,[\dot{\xi}, \eta]\rangle+\langle\alpha,[\xi, \dot{\eta}]\rangle \text {. }
$$

The last two terms vanish by (1.13) and $\dot{\alpha}$ can be any tangent vector in $T W_{\alpha}$. Thus

$$
\left[g_{\alpha}, g_{\alpha}\right] \subset T W_{\alpha}^{0}
$$

By (1.2) this implies that

$$
\left[g_{\alpha}, g_{\alpha}\right] \subset g_{p}
$$

which gives (1.12).

(When we get to discussing integrable systems, we will use the $G_{\alpha}^{0} / G_{p}^{0}$ for the angle variables.)

Let $Z$ be a submanifold of $W$ that is a transverse section to the $G$ orbits of $W$. Thus at each point $\alpha \in W$ we have

$$
T W_{\alpha}=T Z_{\alpha} \oplus T O_{\alpha} \quad \text { (direct sum). }
$$


For any $p \in \Phi^{-1}(\alpha)$ let

Since $U \supset \operatorname{ker} d \Phi_{p}$ we have

$$
U=d \Phi_{p}^{-1}\left(T Z_{\alpha}\right)
$$

and hence

$$
U \supset u_{p}(g)^{\perp}
$$

$$
U^{\perp} \subset u_{p}(g)
$$

We claim that $U$ is a symplectic subspace of $T M_{p}$, i.e., that

$$
U \cap U^{\perp}=\{0\} \text {. }
$$

Indeed, by (1.17) we must show that any $\xi \in g$ satisfying

$$
u_{p}(\xi) \in U^{\perp}
$$

must satisfy $u_{p}(\xi)=0$. But $d \Phi_{p}\left(u_{p}(\xi)\right) \in T O_{\alpha}$ by the equivariance of $\Phi$ and hence if $d \Phi_{p}\left(u_{p}(\xi)\right) \in T Z_{\alpha}$ we must have

$$
d \Phi_{p}\left(u_{p}(\xi)\right)=0 \quad \text { since } T Z_{\alpha} \cap T \mathscr{O}_{\alpha}=\{0\} .
$$

Thus $\xi \in T \mathcal{O}_{\alpha}^{0}$. Also if $u_{p}(\xi) \in U^{\perp}$ then (1.1) implies that $\xi \in T Z_{\alpha}^{0}$. Thus, by (1.16),

$$
\xi \in T W_{\alpha}^{0}=g_{p}
$$

hence $u_{p}(\xi)=0$. Thus we have proved

(1.19). Suppose that $Z$ is a submanifold of $W$ that is a transverse section to the $G$ orbits in the sense that (1.16) holds at all points. Then $\Phi^{-1}(Z)$ is a symplectic submanifold of $M$.

For the case $W=g^{*}$ this was proved in [5]. A particularly interesting case would be when we can choose $Z$ to be a slice in the group theoretic sense. Then the isotropy groups $g_{p}$ at all $\beta \in Z$ would be equal to $g_{\alpha}$. Then, in $\Phi(Z)$ we might be able to choose a slice for the $G$ action, so that all the $g_{p}$ would be equal. (Again this is always possible at general points if $G$ is compact, for example.)

Then we get a Hamiltonian action of $G_{\alpha} / G_{p}$ over $\Phi^{-1}(Z)$, and $G_{\alpha} / G_{p}$ is a torus.

\section{Collective complete integrability}

We now obtain a necessary condition for Thimm's method to work. Suppose that $h_{1}, \ldots, h_{n}$ are functions on $g^{*}$ such that $\Phi^{*} h_{1}, \ldots, \Phi^{*} h_{n}$ are $n$ independent functions which Poisson commute on $M$, where $n=\frac{1}{2} \operatorname{dim} M$. This means that the manifolds

$$
\Phi^{*} h_{1}=\text { constant }, \ldots, \Phi^{*} h_{n}=\text { constant }
$$

are Lagrangian submanifolds of $M$. In particular, for any $p \in M$ with $\Phi(p)=\alpha$ the submanifold $\Phi^{-1}(\alpha)$ is contained in one of the above submanifolds and hence must be isotropic. By (1.6) this implies that

$$
u_{p}(g)^{\perp} \text { is isotropic, }
$$

or, what amounts to the same thing, that

$$
u_{p}(g)^{\perp} \subset u_{p}(g) \text {. }
$$

In other words,

the $G$ orbits are co-isotropic. 
By (1.7), (2.2) is the same as

$$
d \Phi_{p}^{-1}\left(T O_{\alpha}\right)=u_{p}(g)
$$

which, under our cleanness assumptions is the same as

$$
G \text { acts transitively on } \Phi^{-1}\left(\mathscr{O}_{\alpha}\right) \text {. }
$$

This, of course, is the same as saying that

$$
G_{\alpha} \text { acts transitively on } \Phi^{-1}(\alpha) \text {. }
$$

For a general Hamiltonian $G$-manifold the spaces $\Phi^{-1}(\alpha) / G_{\alpha}$ are all symplectic manifolds known as the Marsden-Weinstein reduced spaces, cf. [10]. Then (2.5) is the same as

$$
\text { The Marsden-Weinstein reduced spaces are all points. }
$$

In general, co-istropic $\Phi^{-1}\left(\mathscr{O}_{\alpha}\right)$ quotiented by its null foliation is a symplectic manifold (if it is a manifold) which we may call the KKS reduced space, cf. [7]. Then the preceding are equivalent to

The KKS reduced space of $\mathscr{O}$ is $\mathcal{O}$.

Finally, let us consider the algebra of $G$-invariant functions on $M$. By $\left(2.2^{\prime}\right)$ (generically) the common level surfaces of these functions are co-isotropic. So the $G$-invariant functions Poisson commute. Thus (2.1)-(2.7) are all equivalent to

Condition (2.8). The algebra of all $G$-invariant functions on $M$ is commutative under Poisson bracket.

Condition (2.8) is the 'classical analogue' of a well known 'quantum' property:

If we are given a representation of a group, then the algebra of $G$-invariant operators (those which commute with the $G$ action) is commutative if and only if the representation is multiplicity free, i.e. if and only if every irreducible of $G$ occurs in the representation with multiplicity at most one.

In other words, the quantum action of $G$ is multiplicity free if and only if the algebra of $G$-invariant quantum observables is commutative. The classical analogue would thus be that the algebra of $G$-invariant classical observables be commutative. This is exactly (2.8). For this reason we will call a Hamiltonian $G$-space multiplicity free if any (and hence all) of the equivalent conditions (2.1)-(2.8) hold. (This name was suggested to us by Joe Wolf.) We shall study these multiplicity free spaces in more detail in a forthcoming paper.

We have thus proved:

THEOREM. If the Hamiltonian $G$ space, $M$, admits a collective completely integrable system then it must be multiplicity free.

Now suppose that (2.1)-(2.8) are satisfied at all $p$ and suppose that we are given a foliation $L_{W}$ of $W$ which is tangent to each orbit and Lagrangian when restricted to each orbit. For example we might be given $k$ functions defined on $W$ which Poisson commute and which, when restricted to each orbit, give a Lagrangian foliation. Then $\Phi^{*} L_{W}$ obtained by

$$
\Phi^{*}\left(L_{W}\right)_{p}=d \Phi_{p}^{-1}\left(\left(L_{W}\right)_{\alpha}\right), \quad \alpha=\Phi(p)
$$


defines a Lagrangian foliation of $M$. In particular, if we can find $n-k$ additional functions on $W$ whose common level surfaces are the $G$ orbits on $W$ then we get $n$ functions on $W$ which pull back to give a completely integrable system on $M$.

As we have remarked for $G_{1} \subset G$ the map

$$
\pi^{*}: g^{*} \rightarrow g_{1}^{*}
$$

is just the moment map when restricted to each $G$ orbit. Thus for the Thimm method to work, we must satisfy the criteria (2.1)-(2.8) for the $G_{1}$ action on each $G$ orbit. Proceeding inductively we see that

(2.9). A necessary and sufficient condition for the Thimm method to work is that (2.1)-(2.8) hold for each of the maps

with $g_{n}=\{0\}$.

$$
\Phi: M \rightarrow g^{*}, \quad g^{*} \stackrel{\pi^{*}}{\rightarrow} g_{1}^{*}, \quad g_{1}^{*} \rightarrow g_{2}^{*}, \quad \text { etc }
$$

We shall see in $\$ 4$ that the conditions on the $\pi$ hold generally for the pair

$$
u(n) \supset u(n-1) \text {. }
$$

In fact, this is a result of Heckmann [6] who shows that the same is true for the pair

$$
o(n) \supset o(n-1)
$$

and that these are the only pairs where this holds generically. (In particular it does not hold for the symplectic group pairs. This is why we would expect quaternionic Grassmannians to behave differently from the real or complex ones.)

For any Lie group $G$ there is an alternative method of constructing a family of Poisson commuting functions on $g^{*}$. It consists of taking the $G$-invariant functions on $g^{*}$ and considering all possible shifts

$$
f(\cdot+t a) \quad \text { where } a \text { is in } g^{*} \text { and } t \text { is any real number. }
$$

Mishchenko and Fomenko prove that the collection of these functions all Poisson commute. Furthermore, if $G$ is semi-simple and $a$ is generic, they prove [13] that these provide a completely integrable system on generic orbits. In [1] Dao Chong Tkhi claims to prove that this system is also completely integrable on lower dimensional orbits. But there seem to be some problems with his proof, cf. the comments of Mishchenko in [12]. If the results of [1] are correct, this would imply that every multiplicity free space for a semisimple Lie group has a collective completely integrable system.

Now suppose that we are in the situation described at the end of $\$ 1$, where $Z$ is a slice to the $G$ action on $W$ and $\Phi^{-1}(Z)$ is a symplectic manifold with a Hamiltonian action of the torus $G_{\alpha} / G_{p}$. Let $\Psi$ denote the moment map for this torus action. Then

$$
\operatorname{dim} \Phi^{-1}(Z)=\operatorname{dim} \Psi^{-1}\left[\left(g_{\alpha} / g_{p}\right)\right]^{*} \leq \operatorname{dim}\left(g_{\alpha} / g_{p}\right)+\max \operatorname{dim} \operatorname{ker} d \Psi_{p^{1}} .
$$

Suppose that (2.5) holds. Then

$$
\operatorname{dim} \operatorname{ker} d \Psi_{p^{1}}=\operatorname{dim}\left(g_{\alpha} / g_{p}\right)
$$

so

$$
\operatorname{dim} \Phi^{-1}(Z) \leq 2 \operatorname{dim}\left(g_{\alpha} / g_{p}\right)
$$


But this can only occur, by (2.1), if we have equality everywhere. Thus (2.10). If $Z$ is a slice to the $G$ action on $W$ such that we get a toral action of $G_{\alpha} / G_{p}$ on $\Phi^{-1}(Z)$, and if $(2.5)$ holds, then $G_{\alpha} / G_{p}$ provide the angle variables for a completely integrable system on $\Phi^{-1}(Z)$. In particular, this happens near generic points if $G$ is compact

\section{Cotangent bundles of homogeneous spaces}

Let $X=G / K$ be a homogeneous space and let $M=T^{*} X$. We wish to investigate the meaning of conditions (2.1)-(2.7) for this case. By homogeneity, it suffices to check these conditions at points lying over the base point, $K$.

We may identify $T X_{K}$ with $g / k$ and hence $T^{*} X_{K}$ with $k^{0} \subset g^{*}$. The moment map, when restricted to $T^{*} X_{K}$ is just the injection of $k^{0}$ into $g^{*}$.

To verify (2.4) we may use $G$ acting on $X$ to reduce the verification to $T^{*} X_{K}$ which we have identified with $k^{0}$. Then $K$ still acts on $k^{0}$ and the condition (2.4) says that $K$ must act transitively on

Thus

$$
\Phi^{-1}(\mathcal{O}) \cap T^{*} X_{k}=\mathscr{O} \cap k^{0}
$$

(3.1). For $M=T^{*} X$ with $X=G / K$ the conditions (2.1)-(2.7) are equivalent to the condition :

$$
K \text { acts transitively on } O \cap k^{0} \text {. }
$$

Infinitesimally, this condition says:

(3.2). For each $\xi \in g$ and $l \in k^{0}$,

$$
\xi \cdot l=\eta \cdot l \quad \text { for some } \eta \in k .
$$

For example, if $G$ is the semi-direct product of $K$ with a vector space $V$ (relative to a representation of $K$ on $V$ ) then

$$
T^{*} G / K=T^{*} V \text { and } g^{*}=k^{*}+V^{*} \text {; }
$$

so $k^{\circ}=V^{*}$. The $G$ orbits in $g^{*}$ are described in [3]. The ones which intersect $V^{*}$ are exactly the cotangent bundles of $K \cdot p$ for $p \in V^{*}$ and the intersection is then the zero section of $T^{*} K \cdot p$, i.e. $K \cdot p$ itself, on which $K$ clearly acts transitively. Thus this set-up verifies (3.1).

Thimm considers the situation where there is a non-degenerate invariant scalar product on $g$ which allows the identification of $g^{*}$ with $g$ and the coadjoint representation with the adjoint representation. We may take $p=k^{\perp}$ and identify $k^{0}$ with $p$. Then (3.2) becomes

$$
\{\xi \in p \mid[\xi, l] \in p\}=\{\xi \in p \mid[\xi, l]=[\eta, l] \text { for some } \eta \in k\} .
$$

This condition is certainly satisfied if $[p, p] \subset k$ since both sides of (3.7) are then:

$$
\{\xi \in p \mid[\xi, l]=0\} \text {. }
$$

Thus the conditions are satisfied for the cotangent bundles of compact symmetric spaces.

Another way to see that the equivalent conditions (2.1)-(2.8) are satisfied for symmetric spaces is as follows. Let $G$ be a compact Lie group and $X$ a transitive $G$-space with the property that every irreducible representation of $G$ occurs in 
$L^{2}(X)$ with multiplicity at most one. (This is one of the basic properties of compact symmetric spaces.) Let $U$ be a maximum dimensional orbit of $G$ in $T^{*} X \backslash\{0\}$. We can find $G$-invariant functions

$$
f_{1}, \ldots, f_{N} \quad \text { on } T^{*} X \backslash\{0\}
$$

such that the $d f_{i}$ 's span the normal space to $U$ at every point. Without loss of generality we can further assume that $f_{i}=f_{i}(x, \xi)$ is homogeneous of degree one as a function of $\xi$. Let $P_{i}$ be a pseudodifferential operator with $f_{i}$ as its leading symbol. By averaging over $G$ we can arrange that $P_{i}$ is $G$-invariant. Then, with the assumption above, $P_{i}$ maps each irreducible subspace of $L^{2}(X)$ into itself and is a constant multiple of the identity on this subspace. In particular

$$
\left[P_{i}, P_{j}\right]=0
$$

so

$$
\left\{f_{i}, f_{j}\right\}=0
$$

which implies that $U$ is co-isotropic.

In the next section we shall prove that the Thimm conditions are satisfied for the $U(n)$ and $O(n)$ chains. This then gives Thimm's proof of the complete integrability of the geodesic flow for $U(n)$ and $O(n)$ symmetric spaces. As was mentioned above, if the results of [1] on shifted invariants are correct (or if there is some other way of constructing an appropriate family of Poisson commuting functions on $g^{*}$ ), then this would imply the complete integrability of the geodesic flow for an arbitrary symmetric space. But, at present, this question requires some clarification.

If a homogeneous space $G / K$ has the property that the representation of $G$ on $L^{2}(G / K)$ is multiplicity free, then $(G, K)$ is called a Gelfand pair. We shall prove in a forthcoming paper that $(G, K)$ is a Gelfand pair if and only if the Hamiltonian action of $G$ on $T^{*}(G / K)$ is multiplicity free. Thus $(G, K)$ is a Gelfand pair if and only if $(3.1)$ is satisfied. If $G / K$ is a symmetric space, then $(G, K)$ is a Gelfand pair, but there exist many interesting Gelfand pairs (for example $\left.\left(G_{2}, S U(3)\right)\right)$ which are not symmetric spaces.

The set of all Gelfand pairs $(G, K)$ (with $G$ compact and simple) have been classified by Kramer in [9]. One key step in Kramer's argument leading to his classification is to show that

$$
\operatorname{dim} G \leq 2 \operatorname{dim} K+\operatorname{rank} G,
$$

if $(G, K)$ is a Gelfand pair with $G$ compact and simple. Kramer's proof of this inequality depends upon a heavy use of representation theory. However it can be deduced rather easily from (3.1). We shall give the details in our forthcoming paper.

\section{The Gelfand-Ceitlin construction}

In this section we discuss briefly the case of the chain

$$
u(n) \supset u(n-1) \supset \ldots
$$

Instead of using the Casimirs at each stage, we will find it more convenient to work with the eigenvalues. In the language of slices and cross-sections, we will be looking 
at the intersections of our orbits with the positive Weyl chamber in a maximal torus. Again it is enough to look at one step - the passage from $u(n+1)^{*}$ to $u(n)^{*}$. We may (after dividing by $i$ ) identify these spaces with the spaces of $(n+1) \times(n+1)$ and $n \times n$ hermitian matrices. The projection of

$$
u(n+1)^{*} \stackrel{\pi}{\rightarrow} u(n)^{*}
$$

assigns to each $(n+1) \times(n+1)$ matrix its $n \times n$ lower right corner.

An orbit $\mathcal{O}_{\underline{\alpha}}$ in $u(n+1)^{*}$ is determined by the common eigenvalues of its elements,

$$
\lambda_{0} \leq \lambda_{1} \leq \cdots \leq \lambda_{n}
$$

The minimax principle says that the eigenvalues $\mu$, of $\Phi(A)$ for $A \in \mathcal{O}_{\underline{\Lambda}}$ must intertwine those of $\underline{\lambda}$ :

$$
\lambda_{0} \leq \mu_{1} \leq \lambda_{1} \leq \mu_{2} \leq \lambda_{2} \leq \cdots \leq \mu_{n} \leq \lambda_{n} .
$$

Conversely, every such $A$ occurs in the image of $\pi\left(\sigma_{\lambda}\right)$.

We may pick a cross-section to the $u(n)$ orbits to consist of diagonal matrices with

$$
\mu_{1} \leq \mu_{2} \leq \cdots \leq \mu_{n}
$$

We assume that (4.1) holds and let

$$
\Phi: O_{\lambda} \rightarrow u(n)^{*}
$$

denote the restriction of $\pi$ to $\mathcal{O}_{\lambda}$. Then $\Phi^{-1}(\operatorname{diag} \mu)$ consists of all matrices of the form

$$
\boldsymbol{A}=\left(\begin{array}{cccc}
a_{0} & a_{1} & \cdots & a_{n} \\
\tilde{a}_{1} & \mu_{1} & & 0 \\
\vdots & & \ddots & \\
\bar{a}_{n} & 0 & & \mu_{n}
\end{array}\right)
$$

with eigenvalues $\lambda_{1}, \ldots, \lambda_{n}$. The characteristic polynomial of (4.2) is given by

$$
\prod_{i}\left(\lambda-\mu_{j}\right)\left(\lambda_{0}-a_{0}\right)-\sum\left|a_{i}\right|^{2} \prod_{j \neq i}\left(\lambda-\mu_{j}\right)
$$

For any choice of $\underline{\Lambda}$ and for generic $\underline{\mu}$ in the image of $\Phi\left(\mathcal{O}_{\underline{\Lambda}}\right)$ the values of $a_{0}$ and $\left|a_{i}\right|^{2}, i=1, \ldots, n$, are determined by $\mu$ and the condition that $A$ should lie in $\Phi(\underline{\mu})$. For example, suppose that

$$
\lambda_{0}<\lambda_{1}<\cdots<\lambda_{n} .
$$

Then a generic $\underline{\mu}$ will have

$$
\lambda_{0}<\mu_{1}<\lambda_{2}<\cdots<\mu_{n}<\lambda_{n}
$$

and in particular, no two of the $\mu$ 's equal. Then we can write (4.3) as

$$
\prod_{i}\left(\lambda-\mu_{j}\right)\left[a_{0}-\lambda-\sum \frac{\left|a_{i}\right|^{2}}{\lambda-\mu_{i}}\right] .
$$

(It is clear from (4.6) that if we start with $\underline{\mu}$ and the $a_{i}$ 's then (4.5) must hold for all roots of the characteristic polynomial.) Since the product in front of (4.6) 
does not vanish the condition that we obtain on the $a$ 's is

$$
a_{0}-\lambda_{i}=\sum_{i=1}^{n} \frac{\left|a_{i}\right|^{2}}{\lambda_{j}-\mu_{i}} \quad j=0, \ldots, n .
$$

(This is an equation of intersection of confocal ellipsoids. Notice the close connection with the original computations of Jacobi on geodesic flows on ellipsoids [7] cf. also [14]. Thus the 'little integrable system' of (2.10) is, in this case, intimately related to Jacobi's.)

Now $G_{\underline{\mu}}$ in this case is just the $n$-torus consisting of diagonal matrices and it acts on the $a_{i}$ 's, $i=1, \ldots, n$, by multiplying each $a_{i}$ by an independent phase factor. In particular, it acts transitively on $\Phi^{-1}(\underline{\mu})$ verifying (2.5).

In the case where some of the $\lambda$ 's are equal then (2.5) still holds at generic $\mu$ in $\Phi\left(O_{\underline{\Lambda}}\right)$. The group $G_{\underline{\mu}}$ is larger, but certain $a_{i}$ must vanish and the net effect is as before. For example suppose $n=4$ and

$$
\lambda_{1}=\lambda_{2}=\lambda_{3}<\lambda_{4}<\lambda_{5} .
$$

Then the $\underline{\mu}$ which are generic in $\Phi\left(\mathcal{O}_{\underline{\lambda}}\right)$ will have

$$
\mu_{1}=\mu_{2}=\lambda_{3}<\mu_{3}<\lambda_{4}<\mu_{5}<\lambda_{5} .
$$

Differentiating (4.3) twice and setting $\lambda=\lambda_{1}$ (which is a triple root) gives

$$
\left|a_{1}\right|^{2}=\left|a_{2}\right|^{2}=0 \text {. }
$$

Although $G_{\underline{\mu}}$ contains a $U(2)$ factor, it acts trivially on $\Phi^{-1}(\underline{\mu})$ as expected from $\S 1$. The remaining $a$ 's are determined by equation (4.7) in fewer variables and the left over torus, $G_{\mu} / U(2)$ acts transitively on $\Phi^{-1}(\underline{\mu})$.

Combining the results in this section with those of $\S 3$ proves Thimm's theorem that the geodesic flows on the complex Grassmannians are completely integrable. A similar argument works for the $O(n)$ chain.

We wish to thank the referee and Alan Weinstein for pointing out to us that some of the results in this paper were obtained by Mishchenko [12] and Mikitiuk [11], and calling our attention to related results of Planchart [15].

\section{REFERENCES}

[1] Dao Chong Tkhi. Integrability of Euler's equations on homogeneous symplectic manifolds. Math. Sb. 106 No. 2 (1978), 154-161.

[2] M. Duflo \& M. Vergne. Une propriete de la representation coadjointe d'une algebre de Lie. CRAS Paris Ser. A-B 268 (1969) A583-585.

[3] V. Guillemin \& S. Sternberg. The moment map and collective motion. Ann. of Phys. 127 (1980), 220-253.

[4] V. Guillemin \& S. Sternberg. Convexity properties of the moment map. Inventiones Math. 67 (1982) 491-513.

[5] V. Guillemin \& Sternberg. Moments and reductions. Conference on differential geometric methods in theoretical physics. World Scientific (Singapore) (1983) 87-102.

[6] G. Heckmann. Projections of orbits and asymptotic behavior of multiplicities for compact connected Lie groups. To appear.

[7] C. Jacobi. Vorlesungen uber Mechanik. Gesammelte Abhandlungen. 
[8] D. Kazhdan, B. Kostant \& S. Sternberg. Hamiltonian group actions and dynamical systems of Calogero type. Comm. Pure and App. Math. 31 (1978), 481-507.

[9] M. Kramer. Sphärische Untergruppen in kompakten zusamenhängenden Liegruppen. Compositio Mathematica 38 (1979), 129-153.

[10] J. Marsden \& A. Weinstein. Reduction of symplectic manifolds with symmetry. Reports on Math Phys. 5 (1974), 121-130.

[11] I. V. Mikitiuk. In Dauk Akad. Nauk SSSR 265 (1982) 1074-1078.

[12] A. S. Mishchenko. Integration of the geodesic flows on symmetric spaces. Matem. Zametki 31:2 (1982), 257-262.

[13] A. S. Mishchenko \& A. T. Fomenko. Euler equations on finite dimensional Lie groups. Istvestia 12 (1978).

[14] J. Moser. Various aspects of integrable Hamiltonian systems. Proj. Math 8 Birkhauser, (1980).

[15] A. Planchart. Thesis, Berkeley, 1982.

[16] S. Sternberg. Symplectic Homogeneous spaces. Trans. Amer. Math. Soc. 212 (1975) 113-130.

[17] A. Thimm. Integrable geodesic flows on homogeneous spaces. Ergod. Th. \& Dynam. Sys. 1 (1981), 495-517. 